\title{
2-Amino-7(8)-fluorophenazine $N^{5}, N^{10}$-dioxide: Scale-up and toxicity evaluation
}

Carina Sánchez ${ }^{1}$, Belén Dávila ${ }^{1}$, Marcelo Fernández ${ }^{2}$, Joaquín Afonso ${ }^{2}$, Mirel Cabrera ${ }^{2}$, Pablo Cabral2 ${ }^{2}$, Hugo Cerecetto ${ }^{1,2}$, Nicole Lecot ${ }^{1,2}$, Mercedes González ${ }^{1}$ Grupo de Química Medicinal, Facultad de Ciencias, Universidad de la República, Iguá 4225, Montevideo, 11400, Uruguay.

2Área de Radiofarmacia, Facultad de Ciencias, Universidad de la República, Mataojo s/n, Montevideo, 11400, Uruguay.

\section{INTRODUCTION}

Breast cancer is a solid tumor characterized by a high level of hypoxic areas which are difficult to treat. We have developed bioreductive prodrugs under hypoxic conditions in which 2-amino-7(8)-fluorophenazine $N^{5}, N^{10}$-dioxide (FNZ) stands out as a potent and selective anticancer agent [1-2]. In order to improve the ADME properties FNZ was encapsulated in physiological serum: Tween (PS:T). This work presents the optimization of the synthesis of FNZ, the acute toxicity (Up and Down) and the evaluation of the mutagenic potential in vitro (Ames test) of freeFNZ its encapsulated

\section{OBJECTIVES}

- Improve FNZ the yield to a scale up of $3 \mathrm{~g}$ per batch.

- Evaluate the mutagenic potential of free FNZ and its encapsulated.

- Evaluate the acute toxicity of free FNZ and its encapsulated.

\section{MATERIALS AND METHODS}

Scale-up: The synthesis of FNZ is shown in Scheme 1.

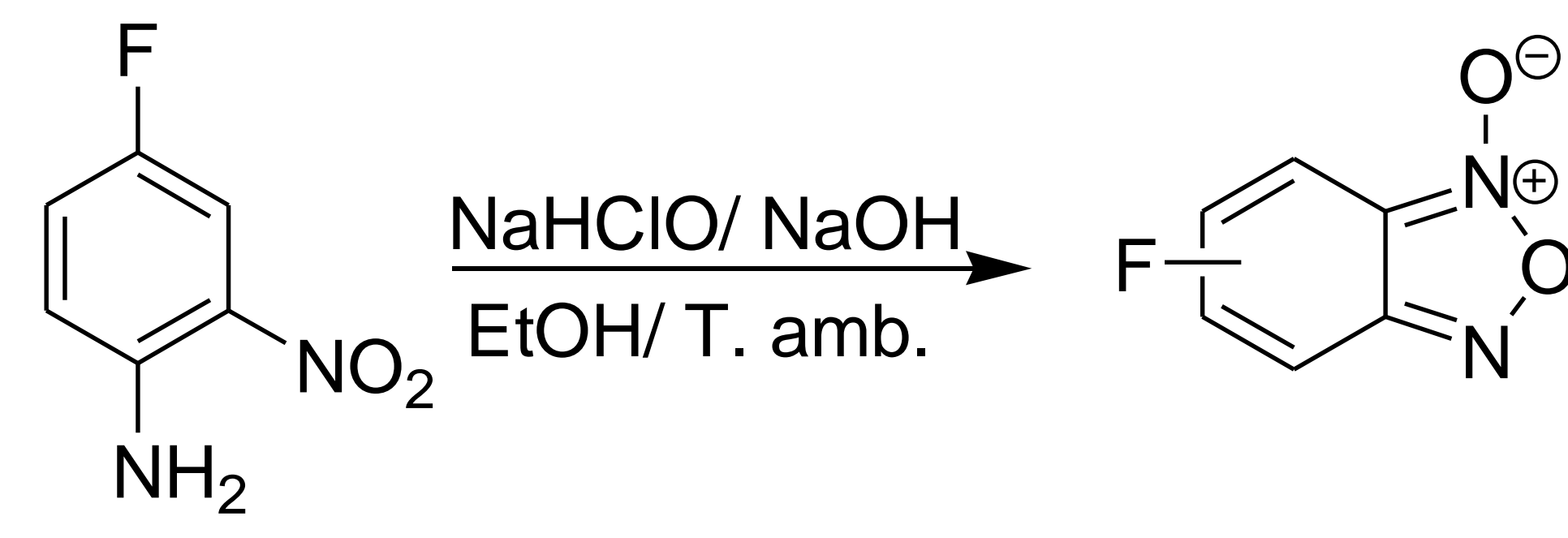

BFX

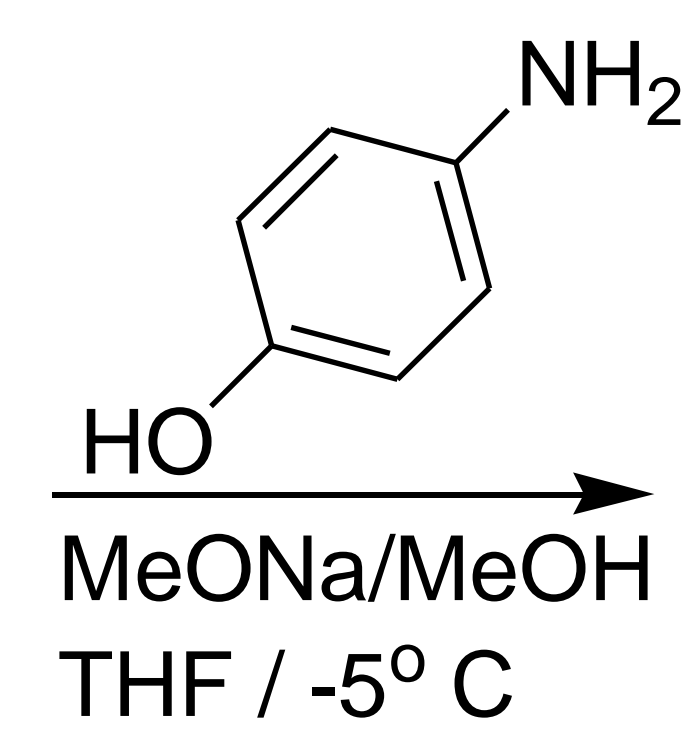

$\mathrm{THF} /-5^{\circ} \mathrm{C}$

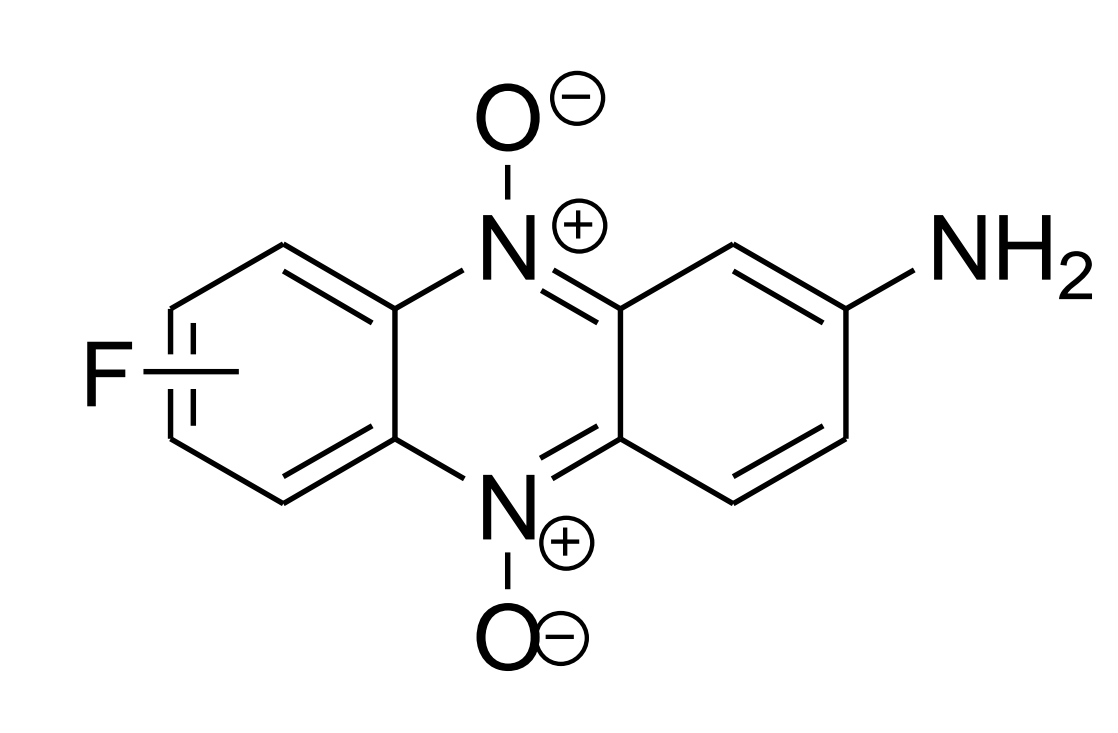

FNZ
Scheme 1. Synthesis of FNZ.

The benzofuroxan intermediate (BFX) was freeze dried for $72 \mathrm{~h}$ (temperature $-52^{\circ} \mathrm{C}$, pressure $1 \mathrm{~Pa}$ ). For the $\mathrm{FNZ}$ synthesis $5 \mathrm{~g}$ of the intermediate (BFX) was used, FNZ was dried in a drying chamber for $5 \mathrm{~d}$, then was washed with $1 \mathrm{~mL}$ of ethyl ether and dried $3 \mathrm{~d}$ in the chamber. Finally, it was freeze dried during $72 \mathrm{~h}$. A dry, red powder was obtained. The structure and purity of FNZ (95\%) was determined by ${ }^{1} \mathrm{H}$ NMR.

Acute toxicity (Up and Down): In this test we studied the acute toxicity of FNZ and its encapsulated in a single peritoneal injection dose equivalent to $2000 \mathrm{mg} / \mathrm{kg}$ of mouse. Particularly, we proved the limit test. BALB/c female mice of 8-12 weeks old were used $(n=5)$ (Figure 1). Mice were monitored for 14 days by recording weights, the first 3 days was controlled parameters contemplated in the Irwin test.

Ames Assay (evaluation of the mutagenic potential): Five strains of Salmonella typhimurium were used to get information about the mutagenicity of FNZ and its encapsulated. The strains of this microorganism are histidine-dependent, so this amino acid is necessary for its growth. The compounds that have the capacity to be mutagenic will be able to reverse this mutation, generating an important growth in a minimal histidine medium overcoming the spontaneous reverts that are given (Figure 2). Positive mutagenicity controls are also used for this assay for each of the strains [4-5].

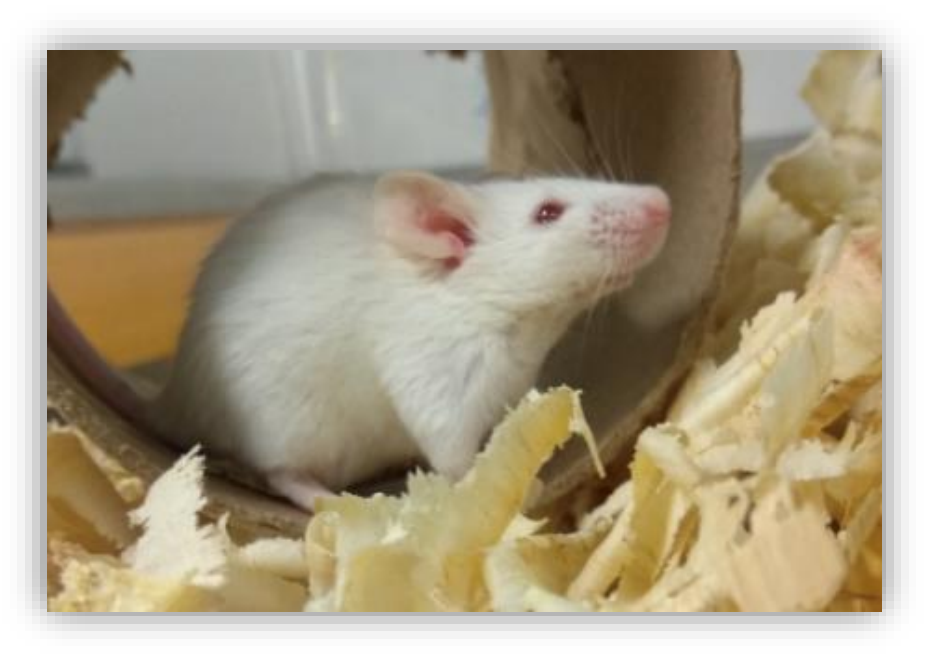

Figure 1. BALB/c female mouse used in Up\&Down test

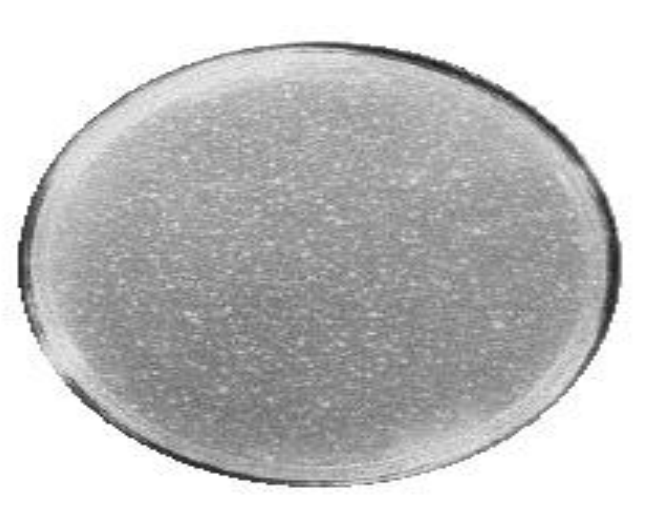

A

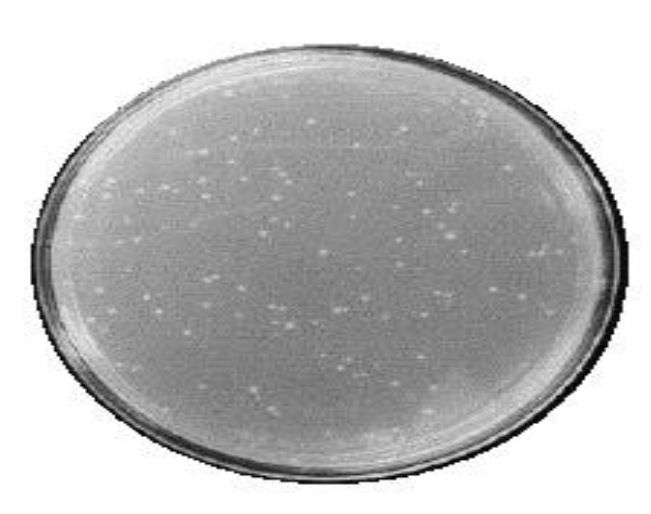

B

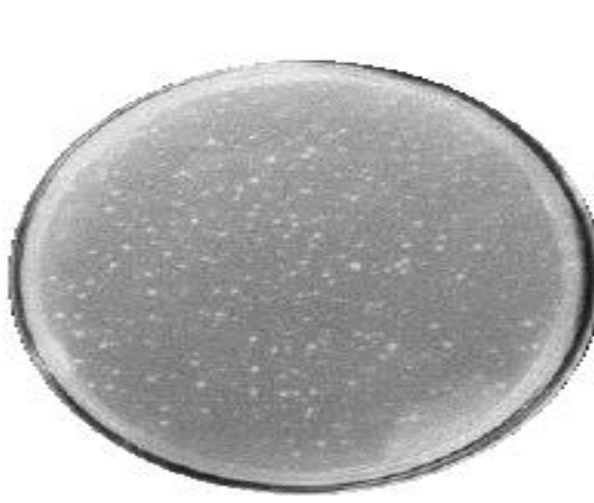

C
Figure 2. Ames assay. A: Positive control, B: Negative control, C: Mutagenic compound

\section{Acknowledgment}

\section{RESULTS}

BFX and FNZ synthesis result in higher yield percentage than the microscale procedure, being the global yield $60 \%$ improving it in more than 13 times the original procedure.

The acute toxicity showed that the encapsulated FNZ has a dose above $2000 \mathrm{mg} / \mathrm{kg}$ of mouse weight and free-FNZ had a maximum dose of $1500 \mathrm{mg} / \mathrm{kg}$ mouse weight. The encapsulated FNZ was not toxic in the Up \& Down assay transforming it in a good formulation to be used in vivo studies.

According to Ames assay the results indicates that all the systems under study (Free-FNZ, PS:T/FNZ) exhibited mutagenicity in the five Salmonella strains. Both systems exhibited mutagenicity and that is why we proceeded to study the mutagenic power of each of them to determine how much their are. The most mutagenic system was the free-FNZ being the encapsulation using (PS:T) the least. As the strains present different mutations we can see that the mutagenic power decreases as the mutation involved is more specific. The specificity decrease from TA 98 to TA 1537 (Figure 3).

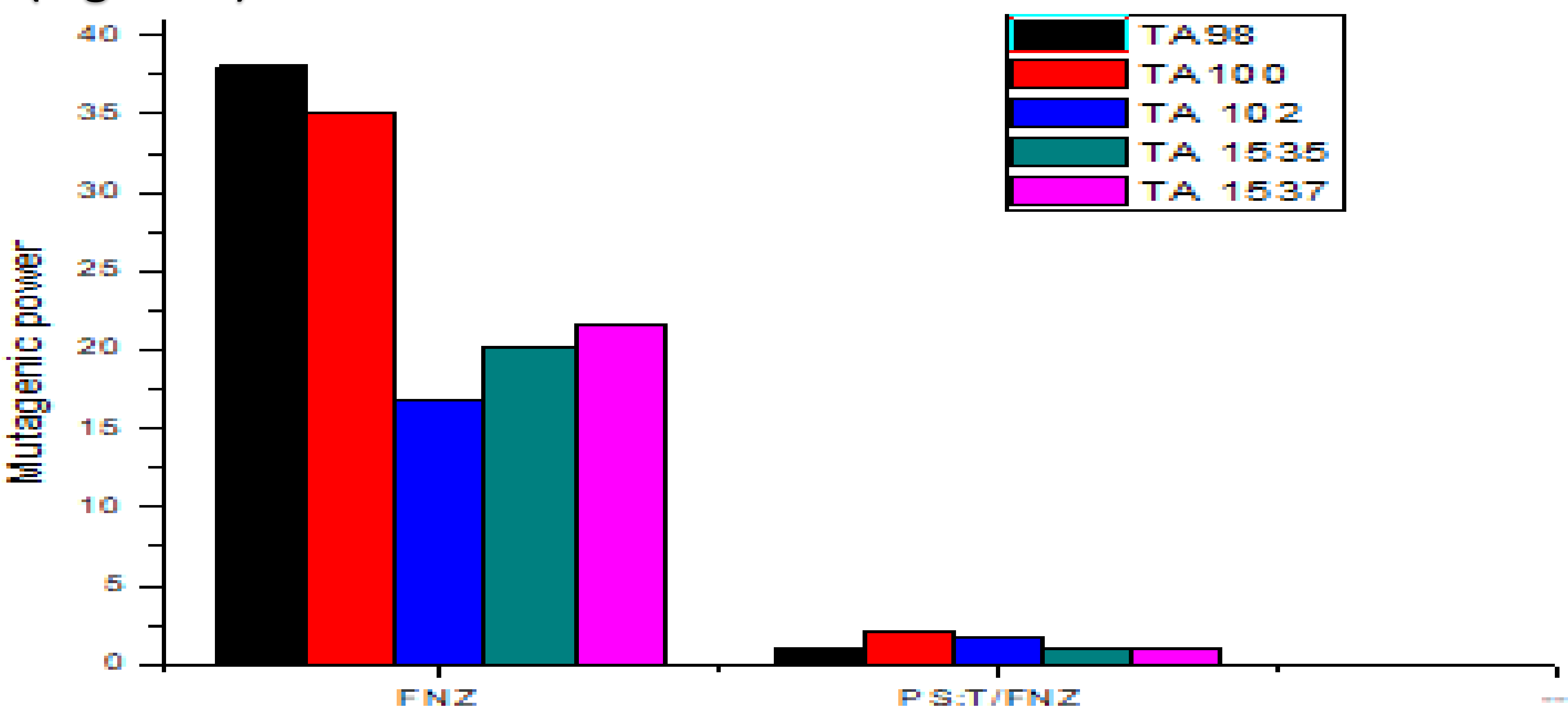

Figure 3. Mutagenic power on the Salmonella strains (TA98, TA100, TA102, TA1535 and TA1537) used in the systems under study.

\section{CONCLUSIONS}

These studies demonstrated that FNZ might be a good drug to be considered for future studies. The synthesis was optimized to obtain better yields and in vivo studies support its low toxicity.

\section{REFERENCES}

[1] Cerecetto $\mathrm{H}$, et al. Phenazine 5, 10-dioxide derivates as hypoxic selective cytotoxins: Part II Structure-activity relationships studies. MedChem. 2006; 5, 511-521.

[2] Cerecetto $\mathrm{H}$, et al. Phenazine 5, 10-dioxide derivates as hypoxic selective cytotoxins: J MedChem.2005;48,21-23.

[3] OECD Guide Acute Oral Toxicity - Up-and-Down-Procedure (UDP).

[4] Maron, D.M; et al. Revised methods for Salmonella mutagenicity test. Mut.Res. 1983;

113, 173-215.

[5] OECD Guide Bacterial Reverse Mutation Test. 